\title{
Fast mapping across time: memory processes support children's retention of learned words
}

\author{
Haley A. Vlach* and Catherine M. Sandhofer \\ Language and Cognitive Development Lab, Department of Psychology, University of California Los Angeles, Los Angeles, CA, USA
}

\section{Edited by:}

Larissa Samuelson, University of

lowa, USA

Reviewed by:

Bradley Love, University College

London, UK

Karla McGregor, University of lowa,

USA

Sarah Kucker, University of lowa, USA

${ }^{*}$ Correspondence:

Haley A. Vlach, Department of

Psychology, University of California

Los Angeles, 1285 Franz Hall, Los

Angeles, CA 90095, USA.

e-mail: haleyvlach@ucla.edu
Children's remarkable ability to map linguistic labels to referents in the world is commonly called fast mapping. The current study examined children's $(N=216)$ and adults' $(N=54)$ retention of fast-mapped words over time (immediately, after a 1-week delay, and after a 1-month delay). The fast mapping literature often characterizes children's retention of words as consistently high across timescales. However, the current study demonstrates that learners forget word mappings at a rapid rate. Moreover, these patterns of forgetting parallel forgetting functions of domain-general memory processes. Memory processes are critical to children's word learning and the role of one such process, forgetting, is discussed in detail - forgetting supports extended mapping by promoting the memory and generalization of words and categories.

Keywords: word learning, fast mapping, memory and learning, long-term memory, forgetting, forgetting curves

\section{INTRODUCTION}

One of the more remarkable developmental feats is the ease by which children appear to learn new words after the second year of life. Children's ability to readily map words to referents in the world and retain these mappings over time, with only minimal exposure, is commonly called fast mapping (e.g., Carey and Bartlett, 1978; see Carey, 2010, for a review). Fast mapping behavior has often been described as having two phases: (1) the initial mapping of a linguistic label to a referent, and (2) the subsequent retention and development of the initial representation, originally termed "extended mapping" (Carey and Bartlett, 1978). The majority of research on fast mapping has focused on cues that children use to initially map words to referents. However, less is known about the processes underlying extended mapping. In this study, we focus our investigation on examining children's retention of fast-mapped words over extended periods of time (i.e., weeks and months) in order to elucidate the memory processes supporting extended mapping and long-term word learning.

\section{FAST MAPPING}

Children are remarkable word learners - they quickly map a novel name onto a referent with one trial or minimal exposure (e.g., Carey and Bartlett, 1978; Heibeck and Markman, 1987; Woodward et al., 1994; Markson and Bloom, 1997; Goodman et al., 1998; Waxman and Booth, 2000; Behrend et al., 2001; Jaswal and Markman, 2001; Wilkinson and Mazzitelli, 2003; Horst and Samuelson, 2008; Carey, 2010; Spiegel and Halberda, 2011). For example, in Carey and Bartlett's (1978) seminal study, preschool-aged children were able to select an olive green tray when their school teacher gestured to two trays, one blue and one olive green, and asked them to get "the chromium tray, not the blue one, the chromium one." After 1 week, children were provided with a brief reminder of the objectlabel mapping and then given a comprehension test. The majority of children at the comprehension test retained the association of "chromium" to the color of olive green. Subsequent studies have documented fast mapping behavior across a variety of categorical domains (e.g., Heibeck and Markman, 1987) and age groups (e.g., Woodward et al., 1994).

Historically, research on fast mapping has also argued that children retain mappings for surprisingly long amounts of time (see Goodman et al., 1998, for a review; Markson and Bloom, 1997; Waxman and Booth, 2000). In fact, some studies have shown that children do not forget words after a week (e.g., Waxman and Booth, 2000) or up to 1 month later (e.g., Carey and Bartlett, 1978; Markson and Bloom, 1997). For example, one study (Waxman and Booth, 2000) presented children with a word learning task in which one of six novel objects was labeled with a novel name. After the single labeling event, children were given an identification and extension test both immediately and after a 1-week delay. All children (100\%) at the 1-week delayed test were able to identify the previously labeled object out of a group of other objects used during the experiment.

In sum, fast mapping behavior includes both the ability to quickly map words to referents in the world and the ability to retain, and build upon, these mappings over time. Although a significant amount of research has focused on the first phase of fast mapping, the initial mapping of words to referents, relatively little research has examined the subsequent processes, such as the retention for these mappings. Consequently, very little is known about the mechanisms underlying children's retention of word mappings. Why do children appear to remember words for extended periods of time? In this study, we examine word learning over time in order to elucidate the memory processes underlying children's retention of words. We ground our investigation of word learning in principles and mechanisms of human memory. Specifically, we contextualize our investigation of word learning in terms of forgetting trajectories, which have been well documented 
by memory research for over 100 years (starting with Ebbinghaus, 1964).

\section{HUMAN MEMORY: THE UBIOUITOUS NATURE OF FORGETTING}

The findings from the fast mapping literature paint the picture that, with only minimal exposure to a word and referent, children know and remember words for extended periods of time. This conclusion is surprising given the large body of memory research that holds the assumption that learned information is forgotten over time. Forgetting is the most ubiquitous characteristic of human memory (see Ebbinghaus, 1964; Shiffrin and Atkinson, 1969; Wickelgren, 1972; Rubin and Wenzel, 1996; Wixted, 2004, for reviews and frameworks of forgetting). Even at a very young age, babies forget information in a similar manner as adults (e.g., Hartshorn et al., 1998; Rovee-Collier et al., 2001).

The natural course of forgetting is described as the relationship between memory and time. As time goes on, the ability to retrieve information declines to a theoretical asymptote of zero. This relationship is commonly characterized by a single mathematical function (e.g., Ebbinghaus, 1964; Wickelgren, 1972; Bahrick, 1984; Loftus, 1985; Anderson and Schooler, 1991; Rubin et al., 1999; Rubin and Wenzel, 1996; White, 2001; Wixted, 2004). Ebbinghaus (1964) was the first to propose a mathematical form of forgetting, a savings function. Since Ebbinghaus (1964) seminal work, researchers have proposed a series of other forgetting functions (see Rubin and Wenzel, 1996; Wixted, 2004; for reviews), including power functions (e.g., Anderson and Schooler, 1991) and exponential-power functions (e.g., White, 2001).

Although forgetting functions may differ on specific dimensions, one similarity across functions is that the rate of forgetting is most rapid initially, but declines as time goes on. That is, forgetting follows a curvilinear pattern, approaching a theoretical asymptote of zero. The curvilinear pattern found in memory tasks is strikingly different than characterizations of word learning performance over time. In particular, fast mapping research commonly characterizes memory for word mappings as a flat, linear pattern over time (e.g., Markson and Bloom, 1997; Goodman et al., 1998; Waxman and Booth, 2000). Because word learning performance has followed a flat, linear pattern, research has concluded that children have high retention of fast-mapped words and do not forget words over time.

\section{DO CHILDREN FORGET FAST-MAPPED WORDS?}

The existing fast mapping literature would suggest that children do not forget newly acquired words over longer periods of time (i.e., weeks and months; e.g., Carey and Bartlett, 1978; Woodward et al., 1994; Goodman et al., 1998; Waxman and Booth, 2000). However, one issue with research on fast mapping is that learning paradigms have commonly been designed to support the high retention of words and prevent forgetting. That is, learning tasks from fast mapping research often incorporate strong memory supports (see Horst and Samuelson, 2008, for a discussion of this issue).

Memory research has long shown that providing memory cues and supports can prevent and/or alter the rate of the forgetting curve by supporting the storage and/or retrieval strength of information (for reviews of these dynamics, see Estes, 1955a,b; Shiffrin and Atkinson, 1969; Wickelgren, 1972; Tulving and Thomson,
1973; Bjork and Bjork, 1992). Examples of such supports include the saliency, repetition, and generation of information. For example, asking participants to generate learned information, during learning, has been show to promote long-term memory to a greater degree than just being presented with information (see Bertsch et al., 2007, for a meta-analysis).

Fast mapping research often incorporates saliency, repetition, and generation into word learning paradigms. In particular, paradigms are often designed so that (1) the labeled object(s) are made more salient than the other objects in the experiment (e.g., Waxman and Booth, 2000), (2) the object is labeled repeatedly, up to nine times (e.g., Woodward et al., 1994), and (3) learners are required to engage in the generation of the mapping and/or word (e.g., Medina et al., 2011). Moreover, many paradigms incorporate a reminder trial immediately preceding the retention test (e.g., Carey and Bartlett, 1978). Thus, the memory supports provided in fast mapping paradigms may be preventing forgetting, and in turn supporting long-term memory for word mappings.

Do children forget fast-mapped words? Because the majority of the existing fast mapping literature has designed word learning paradigms to prevent forgetting, it is unclear what the role of forgetting is in children's long-term word learning. However, recent work has indicated that children may forget fast-mapped words at a rapid rate (Horst and Samuelson, 2008; Horst et al., 2010; Friedrich and Friederici, 2011; Kucker and Samuelson, 2011). For example, Horst and Samuelson (2008) presented 2-year-old children with a referent selection task and a 5-min delayed retention test. Although children initially had high performance when asked to map words to objects, at the referent selection task, children demonstrated very low performance at the 5-min delayed test. This suggests that, when children are required to retain several mappings for just a few minutes, the majority of these mappings are forgotten. In the current study, we built upon this work by demonstrating that older children and adults forget fast-mapped words when required to remember only a single mapping. Moreover, the current experiments were designed to characterize the nature of forgetting over extended periods of time (i.e., weeks and months).

\section{CURRENT STUDY}

In this series of experiments, we sought to demonstrate and characterize the role of forgetting in children's retention of fast-mapped words. To do this, we examined word learning and retention over extended periods of time (i.e., weeks and months) with various degrees of memory support. All participants were presented with a game in which they learned a new word during one of the activities. In Experiment 1, children and adults were not provided with the memory supports commonly presented in fast mapping tasks (i.e., saliency, repetition, and generation). This experiment was designed to determine if forgetting of word mappings would occur in the absence of these supports. In Experiment 2, the experimenter provided children with a varying number of memory supports during the labeling activity.

If forgetting is not a central mechanism underlying fast mapping, children should have an enduring memory for fast-mapped words and exhibit high retention over time. Moreover, children should demonstrate high retention even in the absence of the 
memory supports commonly provided in fast mapping tasks. However, if word learning relies on domain-general memory processes, children should forget words over time, just as memory for other information is forgotten over time. Moreover, this forgetting should be rapid at first and slow with time, just as other information is forgotten in a similar manner (e.g., Rubin and Wenzel, 1996; Wixted, 2004).

\section{GENERAL METHOD}

We adopted our experimental method from Markson and Bloom's (1997) novel word learning paradigm. In this task, learners were presented with a measuring game in which one of the novel objects is casually labeled with a novel word (i.e., "koba"). We chose this paradigm for three reasons. First, this study examined word mapping over extended periods of time, up to 1 month later. Second, this procedure has been used in several investigations of word learning (e.g., Waxman and Booth, 2000). Finally, this study's procedure parallels both seminal research on fast mapping (e.g., Carey and Bartlett, 1978) and more recent research on word learning (e.g., Waxman and Booth, 2000; Behrend et al., 2001).

\section{STIMULI}

In each of the experiments, participants were presented with a measuring game in which they interacted with 10 items, shown in Figure 1. Six of the items were novel objects: a blue plastic tube with a ridged surface, a black sponge-tipped wooden stick, multi-colored trumpet pasta, a gray plastic grid, a brown rubber disk with an indented circle pattern, and a white wooden rectangle consisting of 10 bars connected by 2 longer bars. Children and the vast majority of adults did not spontaneously produce or map familiar words to these objects during the experiment. All of these objects were roughly equivalent in size. Four of the items were familiar objects: a pencil, a ruler, a string, and 10 pennies.

\section{PROCEDURE}

At the beginning of the experiment, children were told they were going to play a game in which they would learn to measure things. Adults were told that they were going to play a game that was

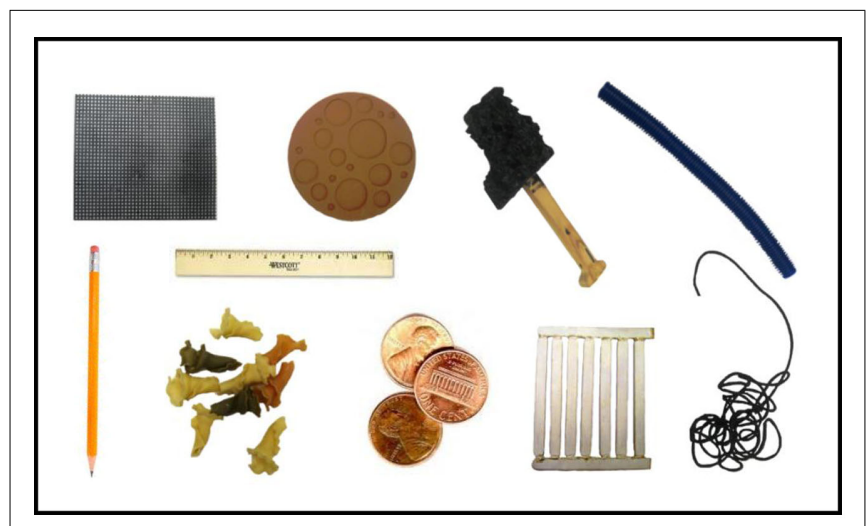

FIGURE 1 | Stimuli used during the experiment. There were four known objects (ruler, pennies, string, and pencil) and six novel objects. Please note that objects are not scaled according to size. designed to teach children how to measure. Participants were not told that they would be participating in a study about word learning. The task was designed to be an incidental word learning task, to model real-world word learning, thus participants were not explicitly told that they would be learning new words.

There were two phases of the experiment: a learning phase and a testing phase. During the learning phase, the experimenter presented participants with six measuring activities. Each of these activities consisted of using one of the familiar objects to measure a novel object. For example, one of the measuring activities was using the ruler to measure the blue tube. The experimenter would bring out both objects simultaneously and say, "Let's measure this toy with this." The experimenter would then have the participant measure the object and say how long it was. After measuring the object, the experimenter would say, "Lets put these away now and play with some other things." The experimenter would then introduce the next measuring activity until all of the measuring activities had been presented.

During one of the six activities, the novel object was casually labeled with a novel word ("koba"). For example, the experimenter would say, "Let's measure the 'koba'." The activity in which this labeling event occurred was randomly assigned for each participant. In all other activities, both the novel and familiar object were not labeled. For example, all other objects were referred to as "this," "it," or "toy." Thus, only one object during the entire learning phase was given a novel label.

The testing phase occurred either immediately, 1 week later, or 1 month later, according to the testing delay condition in which the participant was assigned. Participants were not given a reminder of the initial mapping before the test. The test consisted of the experimenter placing all 10 objects, in random placement order, on the table and asking participants to hand them the "koba." The experimenter at test was a different person than during the learning phase in order to ensure that the experimenter was blind to which object had been labeled the "koba."

\section{EXPERIMENT 1 \\ PARTICIPANTS}

Fifty four 3-year-old children ( $M=43.1$ months, Range: 3648 months, 29 girls) and 54 undergraduate students participated in this experiment. Adult participants received course credit for their participation in the study. Participants in Experiment 1 did not participate in any other experiment in this study. All participants were monolingual English speakers. Children and adults were randomly assigned to one of the three testing delay conditions: immediate, 1 week delay, and 1 month delay. Thus, there were 18 participants in each of the conditions of the experiment.

\section{DESIGN}

This experiment used a 2 (Age Group) $\times 3$ (Testing Delay) design; both the age group (children and adults) and testing delay (immediate, 1 week delay, and 1 month delay) were between-subjects factors.

\section{PROCEDURE}

The procedure used in this experiment was the same as described in the Section "General Methods." In this experiment, children and 


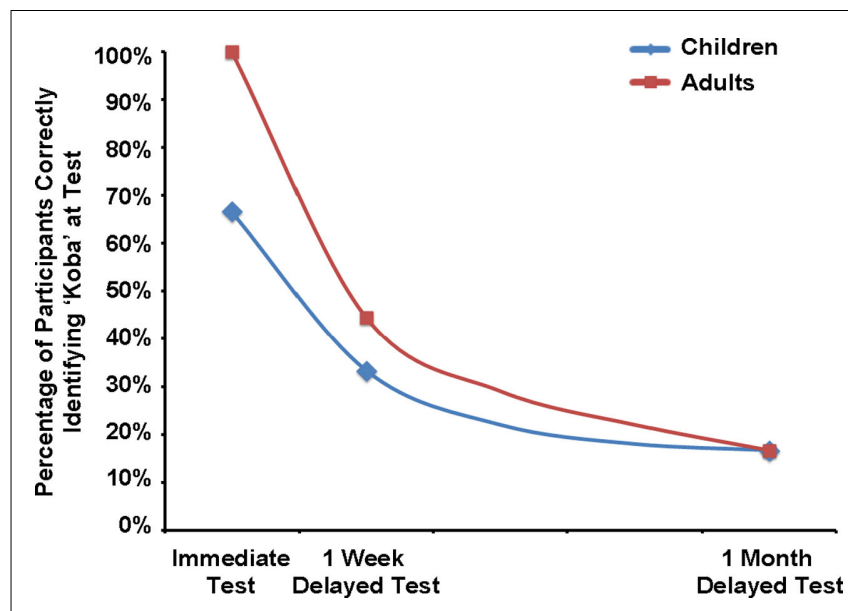

FIGURE 2 | Results of Experiment 1. The percentage of participants (children and adults) correctly identifying the "koba" at the immediate test, 1 week delayed test, and 1 month delayed test. Participants in this experiment did not receive the memory supports typically included in studies of fast mapping and word learning.

adults were not provided with the memory supports typically provided during fast mapping studies. The novel object used during the labeling activity was only casually labeled once ("Let's measure the 'koba'!").

\section{RESULTS AND DISCUSSION}

As can be seen by Figure 2, the percentage of children and adults accurately remembering the mapping of "koba" at test appeared to vary across time. A chi-square analysis confirmed that there were differences in the number of participants successfully and unsuccessfully mapping the label to the object at test across the three testing delay conditions for children, $\chi^{2}(2, N=54)=9.82$, $p=0.0074$, and adults, $\chi^{2}(2, N=54)=26.07, p<0.001$. At the immediate test, most children and nearly all adults were able to correctly map the novel word ("koba") to the object that had been labeled during the learning phase. However, performance at the 1 -week and 1 month test was significantly lower, suggesting forgetting occurred over time. Moreover, the pattern of performance across time appeared to be similar to that of forgetting curves (see Wixted, 2004, for a review) - forgetting followed a curvilinear pattern in which the rate of forgetting was the most rapid initially, but slowed over time.

The results of this experiment suggest that children and adults forget word mappings across time. This is surprising given that previous research has suggested that learners have high retention for fast-mapped words. One possibility is that previous research may have concluded that children do not forget words across time because fast mapping paradigms commonly incorporate strong memory supports (see Horst and Samuelson, 2008, for a discussion). These memory supports may have prevented forgetting, thus giving the appearance of relatively consistent performance across time. We propose that word mapping and memory are intimately related processes. Thus, word mappings, just like other types of learned information, are forgotten over time.
In Experiment 2, we examined word mapping performance when children were provided with additional memory supports during learning. If children's performance varied based upon the number of memory supports present during learning, this suggests that indeed word mapping relies on domain-general memory processes. Moreover, if word mapping performance remained consistent across time, in the presence of memory supports, this provides an explanation for why previous studies have demonstrated high retention over time (e.g., Markson and Bloom, 1997; Goodman et al., 1998; Waxman and Booth, 2000) whereas Experiment 1 and a few other studies have shown that children forget words across time (e.g., Horst and Samuelson, 2008). However, if children's performance was not affected by varying numbers of memory supports during learning, this suggests that domaingeneral memory processes may not support word mapping performance across time. In this case, an alternate explanation is needed to explain why children have an enduring memory for words, but forget other information, and why there are conflicting results in the fast mapping literature.

\section{EXPERIMENT 2}

In this experiment, we sought to determine if varying the memory supports provided to children during learning would affect word mapping performance across time. Because participants forgot word mappings over time in Experiment 1, we proposed that domain-general memory mechanisms are involved in children's long-term word learning. Consequently, we predicted that memory supports would alter the forgetting curves of word mappings.

\section{PARTICIPANTS}

One hundred sixty-two 3-year-old children ( $M=42.8$ months, Range: 36-48 months, 88 girls) participated in this experiment. Participants in Experiment 2 did not participate in any other experiment in this study. All participants were monolingual English speakers. Children were randomly assigned to one of the three memory support conditions: one memory support (saliency), two memory supports (saliency and repetition), or three memory supports (saliency, repetition, and generation); and one of the three testing delay conditions: immediate, 1 week delay, and 1 month delay. In total, there were 54 children in each of the three memory support conditions of this experiment, equally randomly assigned across the three testing delay conditions. Thus, there were a total of 18 participants in each condition of the experiment.

\section{DESIGN}

This experiment used a 3 (Memory Support) $\times 3$ (Testing Delay) design; both the number of memory supports (one, two, and three memory supports during learning) and testing delay (immediate, 1 week delay, and 1 month delay) were between-subjects factors.

\section{PROCEDURE}

During the labeling activity, participants were provided with one, two, or three memory supports: saliency, repetition, and generation. The experimenter provided these supports in the following ways: For saliency, the experimenter made the target object more 
salient by telling the participant that it was special (for example, "This next toy is special.") before it was labeled. For repetition, the experimenter casually labeled the target object repeatedly, for a total of six times (for example, "Let's measure this 'koba." How long is the 'koba'? ...). Finally, for generation, the experimenter asked the participant to generate the word for the target object (for example, "Can you say koba?") immediately before putting the object away. Participants were provided with supports in this manner because this is how they are commonly provided in studies of children's word learning (e.g., Woodward et al., 1994; Markson and Bloom, 1997; Waxman and Booth, 2000; Medina et al., 2011). The rest of the procedure was the same as described in the Section "General Methods."

\section{RESULTS AND DISCUSSION}

As can been seen in Figure 3, the percentage of children accurately remembering the mapping of "koba" at test appeared to vary over time and across the different memory support conditions. We first examined whether children within each memory support condition had varied performance over time. A chi-square analysis confirmed that there were differences in the number of participants successfully and unsuccessfully mapping the label to the object across the three testing delays in the no memory support condition, $\chi^{2}(2, N=54)=9.82, p=0.0074$ (from Experiment $1)$, the one memory support condition, $\chi^{2}(2, N=54)=7.75$, $p=0.0208$, and the two memory supports condition, $\chi^{2}(2$, $N=54)=7.30, p=0.0260$. There was not a significant difference across the three testing delays in the three memory support condition, $\chi^{2}(2, N=54)=1.20, p=0.5488$. Thus, only children in the condition with the most memory supports (the three memory support condition) had high retention over time, without significant forgetting. However, children in all of the other memory support conditions demonstrated forgetting over time.

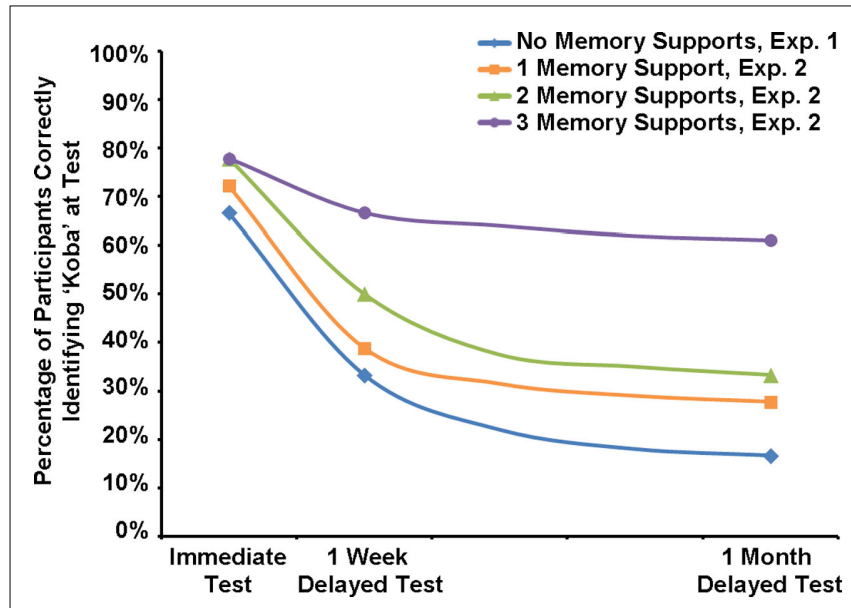

FIGURE 3 | Results of Experiment 2. The percentage of participants (children) correctly identifying the "koba" at the immediate test, 1 week delayed test, and 1 month delayed test, for four conditions of learning: no memory supports (from Experiment 1), one memory support (Experiment 2), two memory supports (Experiment 2), and three memory supports (Experiment 2).
We then examined whether there would be differences in the pattern of forgetting across the four memory support conditions. A chi-square analysis confirmed that there was a difference in the number of participants successfully and unsuccessfully mapping the label to the object across the four memory support conditions at the 1 -month delayed test, $\chi^{2}(3, N=72)=8.52, p=0.0364$. However, there were not significant differences at the immediate test, $\chi^{2}(3, N=72)=0.79, p=0.8519$, and the 1 -week delayed test, $\chi^{2}(3, N=72)=4.68, p=0.1968$. This suggests that, although the effects of the different memory supports were not apparent at the immediate and 1 week delayed test, the memory supports did affect long-term performance at the 1-month test. Because there were differences at the 1-month delayed test, there were also differences in the pattern of forgetting over time. Thus, these results suggest that small changes in an experimental paradigm can alter the manner in which word mappings are remembered and forgotten over time.

The results of Experiment 2 also provide an explanation for why previous studies have suggested that children have high retention of word mappings (e.g., Markson and Bloom, 1997; Goodman et al., 1998; Waxman and Booth, 2000). In studies of fast mapping, experiments are often designed so that they include several memory supports. However, in this study, when these memory supports were removed, children rapidly forgot words over time. This work suggests that, instead of having high retention of words across time, children forget words over time. In the Section "General Discussion," we outline how rapid forgetting may be a critical mechanism underlying children's long-term word learning.

\section{GENERAL DISCUSSION}

For decades, children's ability to quickly map and retain a new word has been described as a remarkable characteristic of language learning and development (e.g., Carey and Bartlett, 1978; Heibeck and Markman, 1987; Goodman et al., 1998; Waxman and Booth, 2000; Behrend et al., 2001). The experiments in this study confirm children's remarkable mapping abilities - the majority of children readily mapped a novel word to a novel object at an immediate test. However, these experiments confirm the findings from recent investigations of fast mapping (e.g., Horst and Samuelson, 2008; Horst et al., 2010; Friedrich and Friederici, 2011; Kucker and Samuelson, 2011) - rather than having high retention of words, children and adults forgot words over time. Indeed, participants' retention of word mappings followed a curvilinear pattern, consistent with the manner in which information is forgotten in memory tasks (e.g., Ebbinghaus, 1964; Anderson and Schooler, 1991; Rubin and Wenzel, 1996; White, 2001; Wixted, 2004).

We contextualize our discussion of these findings in terms of the role of forgetting in word learning. Intuitively, forgetting would seem detrimental to word learning because children would be unable to retrieve word mappings after the initial word learning event. However, we outline how forgetting may be a powerful mechanism supporting extended mapping and long-term word learning - forgetting may support both memory for specific word-to-world mappings and the ability to abstract meaning and generalize words to new experiences. 


\section{FORGETTING PROMOTES EXTENDED MAPPING: MEMORY FOR WORD MAPPINGS}

Forgetting is the most ubiquitous characteristic of human memory (e.g., Rubin and Wenzel, 1996; Wixted, 2004) - after acquiring information, learners begin to forget this information according to a curvilinear pattern over time, toward a theoretical asymptote of zero. Intuitively, forgetting would seem to deter memory because it makes retrieving prior learning more difficult. However, over 100 years of memory research (starting with Ebbinghaus, 1964) have outlined how forgetting is essential for the efficiency and adaptive functioning of memory (see Delaney et al., 2010; Storm, 2011; for recent reviews).

For example, according to study-phase retrieval theories of human memory (see Delaney et al., 2010, for a review; Hintzman and Block, 1973; Johnston and Uhl, 1976; Thios and D'Agostino, 1976), forgetting information in between learning events is critical for the efficiency of memory. Information that is important to remember will likely be presented at a later point in time. Thus, when learners are re-presented with this information, it will be retrieved and get reactivated in memory. The process of retrieving and reactivating a memory results in a slowed rate of future forgetting for this information. Conversely, less important information is not likely to be re-presented to the learner and thus is not retrieved and/or reactivated in the same manner as important information. Consequently, less important information is forgotten at a faster rate than more important information. The end result of these processes is efficiency in memory retrieval - information that is important for us to remember is easily retrieved.

Study-phase retrieval theories may be able to account for how word mappings are retained across time. Although mappings are forgotten in a curvilinear fashion, children are likely to be represented with word mappings at later points in time. Upon subsequent presentations, the mapping(s) will get reactivated in memory. This reactivation slows the rate of future forgetting and, across many presentations, makes word mappings easily retrieved.

This account is consistent with current theories on how word mappings are determined across ambiguous and complex learning situations, such as in cross-situational word learning (e.g., Yu and Smith, 2007; Smith and Yu, 2008). The results of cross-situational word learning research suggest that learners will map linguistic labels to objects that are co-presented at the highest frequency. Interestingly, another bi-product of high co-occurrence mappings is more reactivation in memory than low co-occurrence mappings. Indeed, the processes underlying how word mappings are determined across learning events may engender memory processes that promote the retention of this information (see Vlach and Sandhofer, 2011, for an example).

\section{FORGETTING PROMOTES EXTENDED MAPPING: GENERALIZATION OF WORDS}

The process of extended mapping includes more than just the retention of word mappings - extended mapping also includes the development of the initial representation (Carey and Bartlett, 1978). One important component of extended mapping is the ability to accurately extend and/or generalize a word to a novel referent (e.g., Carey and Bartlett, 1978; Waxman and Booth, 2000; Behrend et al., 2001; Jaswal and Markman, 2003; Wilkinson and
Mazzitelli, 2003). Recently, research on forgetting has been contextualized in generalization tasks (e.g., Kornell and Bjork, 2008; Vlach et al., 2008, 2012; Kornell et al., 2010). Generalization tasks differ from memory tasks in that, instead of being asked to recall a specific piece of information from memory, learners are required to abstract across variable learning experiences in order to generalize information to a new situation. This body of work has revealed that providing learners the opportunity to forget information in between learning events supports the acquisition and generalization of knowledge.

One domain in which forgetting has been identified to be particularly important is children's generalization of words and categories (e.g., Vlach et al., 2008, 2012). As an example, one study (Vlach et al., 2008) presented children with a novel noun generalization task on two learning schedules, massed and spaced. In this task, children were presented with exemplars of a novel object category which were labeled with a common novel word (e.g., "blicket"). In the massed presentation schedule, category exemplars were presented in immediate succession (i.e., one right after the other). In the spaced presentation schedule, category exemplars were distributed in time by $30 \mathrm{~s}$ (i.e., $30 \mathrm{~s}$ of time in between each presentation). The 30-s interval in the spaced schedule provided learners the opportunity to forget information in between learning events. Results of the study revealed that, at a 3-min delayed generalization test, children had higher performance on the test for categories presented on a spaced schedule than categories presented on a massed schedule. This finding suggests that providing children the opportunity to forget information during learning promoted their ability to generalize words to novel category exemplars.

Research has suggested that forgetting promotes word learning because it supports the abstraction, and subsequent generalization, of information (e.g., Vlach et al., 2008, 2012). By this account (an extension of study-phase retrieval theory; e.g., Delaney et al., 2010), forgetting promotes abstraction by supporting the memory of relevant features of a category and deterring the memory of irrelevant features of a category. Relevant features of a category are likely to be present at multiple learning events, thus reactivated in memory. This reactivation not only increases the retrieval strength for relevant features, but slows the future forgetting rate of the relevant features. On the other hand, irrelevant features are less likely to be present at multiple learning events, thus not being reactivated in memory. Because irrelevant features are not reactivated, they continue to be forgotten at a faster rate than relevant features. Consequently, when learners are required to generalize information at a future point in time, they will successfully recall more relevant features than irrelevant features, promoting more successful generalization. Indeed, extended mapping includes both successful retention and generalization and forgetting may be simultaneously supporting both processes.

\section{FUTURE RESEARCH: WORD LEARNING AND MEMORY}

This work contributes to a growing body of research demonstrating the intimate relationship between word learning and memory (e.g., Samuelson and Smith, 1998; Smith, 2002; Sandhofer and Doumas, 2008; Vlach et al., 2008; McGregor et al., 2009). The current study highlights a powerful memory process underlying 
word mapping - forgetting - and is the first to demonstrate that children's retention of word mappings follow the same pattern as forgetting functions. It is important to build a mechanistic model for how words are learned and retained rather than just assume all mappings are retained for extended periods of time (see Mayor and Plunkett, 2010; for a model which demonstrates the importance of discarded mappings). Thus, future work should continue to investigate the role of memory processes involved in children's word learning.

Research on fast mapping may also benefit from examining the short-term memory processes involved in word mapping. Shortterm memory processes may be mediating and/or moderating children's use of environmental cues that guide the in-the-moment

\section{REFERENCES}

Anderson, J. R., and Schooler, L. J. (1991). Reflections of the environment in memory. Psychol. Sci. 2, 396-408.

Bahrick, H. P. (1984). Semantic memory content in permastore: fifty years of memory for Spanish learned in school. J. Exp. Psychol. Gen. 113, $1-29$.

Behrend, D. A., Scofield, J. A., and Kleinknecht, E. (2001). Beyond fast mapping: young children's extensions of novel words and novel facts. Dev. Psychol. 37, 698-705.

Bertsch, S., Pesta, B. J., Wiscott, R., and McDaniel, M. A. (2007). The generation effect: a meta-analytic review. Mem. Cognit. 35, 201-210.

Bjork, R. A., and Bjork, E. L. (1992). "A new theory of disuse and an old theory of stimulus fluctuation," in From Learning Processes to Cognitive Processes: Essays in Honor of William K. Estes, Vol. 2, eds A. Healy, S. Kosslyn, and R. Shiffrin (Hillsdale: Erlbaum), 35-67.

Carey, S. (2010). Beyond fast mapping. Lang. Learn. Dev. 6, 184-205.

Carey, S., and Bartlett, E. (1978). Acquiring a single new word. Proc. Stanford Child Lang. Conf. 15, 17-29.

Delaney, P. F., Verkoeijen, P. J., and Spirgel, A. (2010). Spacing and testing effects: a deeply critical, lengthy, and at times discursive review of the literature. Psychol. Learn. Motiv. Adv. Res. Theory 53, 63-147.

Ebbinghaus, H. (1964). Memory: A Contribution to Experimental Psychology, trans. H. A. Ruger and C. E. Bussenius. New York: Dover Publications. [Original work published in 1885].

Estes, W. K. (1955a). Statistical theory of distributional phenomenon in learning. Psychol. Rev. 62, 369-377.

Estes, W. K. (1955b). Statistical theory of spontaneous regression and recovery. Psychol. Rev. 62, 145-154.

Friedrich, M., and Friederici, A. D. (2011). Word learning in 6-month-olds: fast encoding - weak retention. J. Cogn. Neurosci. 23, 3228-3240.

Goodman, J. C., McDonough, L., and Brown, N. B. (1998). The role of semantic context and memory in the acquisition of novel nouns. Child Dev. 69, 1330-1344.

Hartshorn, K, Rovee-Collier, C., Gerhardstein, P. C., Bhatt, R. S., Klein, P. J., Aaron, F., Wondoloski, T. L., and Wurtzel, N. (1998). Developmental changes in the specificity of memory over the first year of life. Dev. Psychobiol. 33, 61-78.

Heibeck, T., and Markman, E. (1987). Word learning in children: an examination of fast mapping. Child Dev. 58, 1021-1034.

Hintzman, D. L., and Block, R. A. (1973). Memory for the spacing of repetitions. J. Exp. Psychol. 99, 70-74.

Horst, J. S., and Samuelson, L. K. (2008). Fast mapping but poor retention in 24-month-old infants. Infancy 13, 128-157.

Horst, J. S., Scott, E. J., and Pollard, J. A. (2010). The role of competition in word learning via referent selection. Dev. Sci. 13, 706-713.

Jaswal, V. K., and Markman, E. M. (2001). Learning proper and common names in inferential versus ostensive contexts. Child Dev. 72, 768-786.

Jaswal, V. K., and Markman, E. M. (2003). The relative strengths of indirect and direct word learning. Dev. Psychol. 39, 745-760.

Johnston, W. A., and Uhl, C. N. (1976). The contributions of encoding effort and variability to the spacing effect on free recall. J. Exp. Psychol. Hum. Learn. Mem. 2, 153-160.

Kornell, N., and Bjork, R. A. (2008). is spacing the enemy of induction? Psychol. Sci. 19, 585-592.

Kornell, N., Castel, A. D., Eich, T. S., and Bjork, R. A. (2010). Spacing Learning concepts and categories:

mapping of a word to an object. Indeed, discovering the memory processes supporting both the initial mapping and retention of words is likely to elucidate the mechanisms of children's word learning over time and across development.

\section{ACKNOWLEDGMENTS}

We thank the three reviewers for their feedback on this paper. We also thank the undergraduate research assistants of the Language and Cognitive Development Lab for their contribution to this project. Furthermore, we appreciate all of the help from the preschool staff, parents, and children that participated in this study. The research in this paper was supported by NICHD grant R03 HD064909-01.

as the friend of both memory and induction in younger and older adults. Psychol. Aging 25, 498-503.

Kucker, S. C., and Samuelson, L. K. (2011). The first slow step: differential effects of object and wordform familiarization on retention of fast-mapped words. Infancy. doi: 10.1111/j.1532-7078.2011.00081.x

Loftus, G. R. (1985). Evaluating forgetting curves. J. Exp. Psychol. Learn. Mem. Cogn. 11, 397-406.

Markson, L., and Bloom, P. (1997). Evidence against a dedicated system for word learning in children. Nature 385, 813-815

Mayor, J., and Plunkett, K. (2010). A neurocomputational account of taxonomic responding and fast mapping in early word learning. Psychol. Rev. 117, 1-31.

McGregor, K. K., Rohlfing, K. J., Bean, A., and Marschner, El. (2009). Gesture support for word learning: the case of under*. J. Child Lang. 36 807-828.

Medina, T. N., Snedeker, J., Trueswell, J. C., and Gleitman, L. R. (2011). How words can and cannot be learned by observation. Proc. Natl. Acad. Sci. U.S.A. 108, 9014-9019.

Rovee-Collier, C., Hayne, H., and Colombo, M. (2001). The Development of Implicit and Explicit Memory. Amsterdam: John Benjamins.

Rubin, D. C., Hinton, S., and Wenzel, A. (1999). A precise time course of retention. J. Exp. Psychol. Learn. Mem. Cogn. 25, 1161-1176.

Rubin, D. C., and Wenzel, A. E. (1996). One hundred years of forgetting: a quantitative description of retention. Psychol. Rev. 103, 734-760.

Samuelson, L. K., and Smith, L. B. (1998). Memory and attention make smart word learning: an alternative account of Akhtar, Carpenter, and Tomasello. Child Dev. 69, 94-104.

Sandhofer, C. M., and Doumas, L. A. A. (2008). Order of presentation effects in learning color categories. J. Cogn. Dev. 9, 194-221.

Shiffrin, R. M., and Atkinson, R. C. (1969). Storage and retrieval processes in long-term memory. Psychol. Rev. 76, 179-193.

Smith, L. B. (2002). "Learning how to learn words: an associate crane," in Becoming a Word Learner: A Debate on Lexical Acquisition, eds R. M. Golinkoff, K. Hirsh-Pasek, L. Bloom, L. Smith, A. Woodward, N. Akhtar, M. Tomasello, and G. Hollich (New York: Oxford University Press), 51-80.

Smith, L. B., and Yu, C. (2008). Infants rapidly learn word-referent mappings via cross-situational statistics. Cognition 106, 1558-1568.

Spiegel, C., and Halberda, J. (2011). Rapid fast-mapping abilities in 2 year-olds. J. Exp. Child Psychol. 109, 132-140.

Storm, B. C. (2011). The benefit of forgetting in thinking and remembering. Curr. Dir. Psychol. Sci. 20, 291-295.

Thios, S. J., and D'Agostino, P. R. (1976). Effects of repetition as a function of study-phase retrieval. J. Verbal Learn. Verbal Behav. 15, 529-536.

Tulving, E., and Thomson, D. M. (1973). Encoding specificity and retrieval processes in episodic memory. Psychol. Rev. 80, 352-373.

Vlach, H. A., Ankowski, A. A., and Sandhofer, C. M. (2012). At the same time or apart in time? The role of presentation timing and retrieval dynamics in generalization. J. Exp. Psychol. Learn. Mem. Cogn. 38, 246-254.

Vlach, H. A., and Sandhofer, C. M. (2011). "Retrieval dynamics of inthe-moment and long-term statistical word learning," in Proceedings of the 33rd Annual Conference of the Cognitive Science Society, eds L. Carlson, C. Hölscher, and T. Shipley (Boston: Cognitive Science Society), 789-794. 
Vlach, H. A., Sandhofer, C. M., and Kornell, N. (2008). The spacing effect in children's memory and category induction. Cognition 109, 163-167.

Waxman, S. R., and Booth, A. E. (2000). Principles that are invoked in the acquisition of words, but not facts. Cognition 77, B33-B43.

White, K. G. (2001). Forgetting functions. Anim. Learn. Behav. 29, 193-207.

Wickelgren, W. A. (1972). Trace resistance and the decay of long-term memory. J. Math. Psychol. 9, 418-455.
Wilkinson, K. M., and Mazzitelli, K. (2003). The effect of 'missing' information on children's retention of fast-mapped labels. J. Child Lang. $30,47-73$.

Wixted, J. T. (2004). The psychology and neuroscience of forgetting. Annu. Rev. Psychol. 55, 235-269.

Woodward, A. L., Markman, E. M., and Fitzsimmons, C. M. (1994). Rapid word learning in 13- and 18-month-olds. Dev. Psychol. 30, 553-566.

Yu, C., and Smith, L. B. (2007). Rapid word learning under uncertainty via cross-situational statistics. Psychol. Sci. 18, 414-420.

Conflict of Interest Statement: The authors declare that the research was conducted in the absence of any commercial or financial relationships that could be construed as a potential conflict of interest.

Received: 13 October 2011; accepted: 08 February 2012; published online: 27 February 2012.

Citation: Vlach HA and Sandhofer CM (2012) Fast mapping across time: memory processes support children's retention of learned words. Front. Psychology 3:46. doi: 10.3389/fpsyg.2012.00046

This article was submitted to Frontiers in Developmental Psychology, a specialty of Frontiers in Psychology.

Copyright () 2012 Vlach and Sandhofer. This is an open-access article distributed under the terms of the Creative Commons Attribution Non Commercial License, which permits non-commercial use, distribution, and reproduction in other forums, provided the original authors and source are credited. 